\title{
Title: Time is vision in recurrent optic neuritis
}

\author{
Authors: Esther Osinga ${ }^{1,2}$, Bob van Oosten ${ }^{1}$, Willemien de Vries-Knoppert ${ }^{2}$, Axel Petzold ${ }^{1,2,3}$
}

Affiliations: 1 Department of Neurology, VUmc MS Centre Amsterdam, NL

2 Expertise Centre Neuro-ophthalmology, VUmc, Amsterdam, NL

3 Moorfields Eye Hospital, The National Hospital for Neurology and Neurosurgery \& UCL Institute of Neurology, Queen Square, London, UK

Corresponding Author: Dr Axel Petzold

UCL Institute of Neurology, Queen Square

London, WC1N 3BG, UK; email: a.petzold@ucl.ac.uk

\section{Contributions}

All authors contributed to the design of the study. EO collected and analysed the data and wrote the first draft of the manuscript. EO and AP have revised the draft. All authors have reviewed and edited the final version of the manuscript.

\section{Declaration of interest}

AP reports that the VUmc MS Centre Amsterdam participated in the OCTIMS trial and the centre has received research support for OCT projects from the Dutch MS Society. The research of AP was supported by the National Institute for Health Research (NIHR) Biomedical Research Centre based at Moorfields Eye Hospital NHS

Foundation Trust and UCL Institute of Ophthalmology. The views expressed are those of the author(s) and not necessarily those of the NHS, the NIHR or the Department of Health.

\section{Acknowledgements}

The authors acknowledge supported by the University College London Comprehensive Bio-medical Research Centre, the Moorfields Biomedical Research Centre, the Dutch MS Research Foundation and ERN-EYE. We thank the referee for suggesting the subgroup analyses.

\section{Running title: Time is vision}




\section{ABSTRACT}

In optic neuritis (ON) inflammation precedes onset of demyelination and axonal loss. The antiinflammatory properties of corticosteroids may be most effective in the early inflammatory phase, but rapid patient recruitment remains a logistic challenge. The aim of the study was to review the effect of time to initiation of treatment on visual outcome in recurrent ON.

A retrospective case note review of patients known to our centre with recurrent $\mathrm{ON}$. The primary clinical outcome was change of best corrected high contrast visual acuity (BCVA). The secondary outcome was the change of optical coherence tomography (OCT) thickness of the peripapillary retinal nerve fibre layer ( $p R N F L$ ) and macular ganglion cell layer (mGCL) from baseline and after a minimum of 3 months following the episode of recurrent ON.

Of 269 patients with a previous episode of ON, 54 experienced recurrent ON. In total 40 OCT documented episodes of relapsing ON were captured in 19 patients. Treatment within $<2$ days led to better recovery of the BCVA $(+0.02)$ and $\mathrm{mGCL}(-2.4 \mu \mathrm{m})$ if compared to delayed treatment (BCVA -0.2 , $p=0.036, m G C L-25.6 \mu m, p=0.019$ ) or no corticosteroids treatment (BCVA $-0.2, p=0.045, G C L-5.0 \mu m$, $p=0.836)$.

These data suggest a beneficial effect of hyperacute corticosteroid treatment. A pragmatic approach for a prospective treatment trial should consider patients with recurrent ON for logistic reasons. 
Keywords:

Recurrent Optic Neuritis, Corticosteroids, Visual Acuity, Optical Coherence Tomography 


\section{Introduction}

The North American optic neuritis treatment trial (ONTT) has influenced the medical management of optic neuritis (ON) [Group \& others 1991; Beck et al. 1992a] . Over the past 25 years new developments have changed the clinical spectrum and diagnostic work up. Whilst testing for syphilis was mandatory in 1992 [Beck et al. 1992b] , contemporary diagnostic work up includes testing for autoantibodies such to aquaporin 4 (AQP4) and myelin glycoprotein (MOG) [Asgari,Owens,Frøkiaer,Stenager,Lillevang \& Kyvik 2011; Petzold et al. 2014] . In addition, retinal axonal degeneration has been established as a key pathological feature quantifiable by retinal optical coherence tomography (OCT), a technique not included to the ONTT [Petzold et al. 2010] . One conclusion of the ONTT, superiority of intravenous compared to oral corticosteroid treatment, has been refuted by a recent non-inferiority trial [Le Page et al. 2015] . In view of these new developments it is worthwhile to remember that the failure of the ONTT to include any pharmacokinetic data and the lack of a biological plausible explanation to why oral corticosteroids should be harmful compared to intravenous corticosteroids had already been debated in 1992 [Achiron,Djaldetti \& Ziv 1992; Moskopp 1992] .

Experimentally, inflammation of the optic nerve precedes demyelination and axonal degeneration by two days [Shindler,Ventura,Dutt \& Rostami 2008]. Irreversible damage to the axonal cytoskeleton will occur within 5-7 days [Zhu et al. 1999]. Therefore the time window for successful treatment initiation is narrow. Corticosteroids have anti-inflammatory and neuroprotective properties [Rosenberg-Schaffer \& Lucas 1993; Banik,Matzelle,Terry \& Hogan 
1997; Sapolsky,Romero \& Munck 2000] . Targeting the inflammatory phase of optic neuritis requires rapid patient recruitment, a logistic challenge for the ONTT and all subsequent ON trials [Petzold 2017] .Recurrent episodes of ON occur frequently in NMO and CRION, but also affect about $5.5 \%$ of patients with multiple sclerosis (MS) [Nikoskelainen 1975; Burman,Raininko \& Fagius 2011; Petzold et al. 2014] .

Therefore is interesting to note that two studies demonstrated hyperacute corticosteroid treatment to improve outcome in patients with Chronic Relapsing Inflammatory Optic Neuropathy (CRION) and neuromyelitis optica (NMO) [Nakamura et al. 2010; Plant,Sibtain \& Thomas 2011]. Here we review the outcome of this practise in patients with recurrent ON for (1) speed of treatment initiation, (2) outcome of visual acuity and (3) outcome of retinal layer atrophy.

\section{Results}

The inclusion and exclusion of patients from the hospital database to our study is summarised as a flow-chart (Figure 1).

The hospital database had 269 patients registered with ON. Of these OCT scans were recorded in 101. Revision of these 101 patients revealed that 47 did not have a diagnosis of optic neuritis according to diagnostic criteria [Petzold et al. 2014] . Of the remaining 54 patients ON was 
monophasic in 35. Taken together 19 patients experienced recurrent episodes of ON whilst under our care. These 19 patients had a total of 40 episodes of OCT documented recurrent episodes of ON.

The median age of these 19 patients was 31 years (IQR 26-44). The baseline characteristics are presented in Table 1. A majority of these patients suffered from RION ( $n=9)$, followed by MSON (n=4), CRION (n=4) and NMO-ON (n=2).

The clinical subgroups were comparable for age and gender. As expected, patients with RION, CRION and NMO-ON had more recurrent episodes of ON compared to MSON (Table 2).

The median interval of onset of symptoms and administration of corticosteroids was one day (IQR 1-5 days). Figure 2 summarises the timing of episodes and treatment initiation per patient. In total, 14 episodes were treated within two days, 15 episodes received delayed treatment and 11 episodes were not treated with corticosteroids. The proportional breakdown of treatment episodes per clinical group was summarised in table 3. Most of the patients with MSON did not received corticosteroids. In contrast all of the patients with CRION did.

\section{Primary outcome measure: BCVA}

\section{Hyperacute corticosteroid treatment compared to no treatment}

Hyperacute corticosteroid treatment initiated within two days was related with a significant better visual outcome $(\mathrm{BCVA}+0.02)$ in eyes with recurrent $\mathrm{ON}(\mathrm{n}=36)$ compared to eyes with recurrent $\mathrm{ON}$ which were not treated (BCVA -0.2, p=0.045, Figure 3). 
Hyperacute compared to delayed corticosteroid treatment

Hyperacute treatment started within two days after symptom onset leads to a significant better BCVA compared to delayed treatment initiation ( $\mathrm{p}=0.036$, Figure 3). The subgroup analyses showed also that the primary outcome remained significantly better comparing treatment initiation within three days, four days, five days or six days compared to delayed initiation (Supplementary Figure A-D, $\mathrm{p}<0.05$ for all comparisons). Significance was lost after further delay (six days, Supplementary Figure D). Likewise a treatment delay of up 14 days, as permitted in the ONTT, did not improve on final BCVA (Supplementary Figure E).

\section{Secondary outcome measure: OCT}

In total, 70 OCT scans were analysed. Automated layer segmentation was successful in all Bscans and none required manual correction. Because of recurrent episodes affecting either eye only 21 episodes had appropriately timed OCT scans eligible for analyses. The change of thickness of the pRNFL and mGCL before and after recurrent episodes ON are shown in Figure 4.

Microcystic Macular Oedema (MMO) was not noted in the OCT scans reviewed. There was significant preservation of the mGCL for episodes treated hyperacutely with corticosteroids (2.4 $\mu \mathrm{m}$ atrophy, $\mathrm{n}=12)$ if compared to patients receiving delayed treatment ( $25.6 \mu \mathrm{m}$ atrophy, $\mathrm{p}=0.019, \mathrm{n}=7$, Figure 4). Consistent with the mGCL findings a larger degree of $\mathrm{pRNFL}$ atrophy 
was also seen with delayed treatment initiation if compared to hyperacute treatment initiation, but this just failed to reach statistical significance ( $\mathrm{p}=-0.08$, Figure 4$)$. OCT data were only available from 3 episodes of relapsing $\mathrm{ON}$ from patients who did not receive any treatment which failed to reach a statistical significance for comparisons of the pRNFL and mGCL with either treatment group.

\section{Subgroup analyses}

In addition to the pooled data analysis subgroups were investigated by pathology in descending group size (1) RION ( $n=9)$, MSON ( $n=4)$, CRION ( $n=4)$, NMO-ON ( $n=2)$ for treatment initiation within two days, delayed or no treatment.

\section{RION}

The subgroup analysis of patients with RION demonstrated a significant better primary outcome for hyperacute corticosteroid treatment in the affected eyes $(\triangle \mathrm{BCVA}+0.1)$ if compared to the untreated group ( $\triangle \mathrm{BCVA}-0.2, \mathrm{p}=0.013)$. Statistical significance was not reached for the secondary outcome measure.

\section{MSON}

In the subgroup analysis for patients with MSON there was no statistical significant differences between patients with hyperacute corticosteroid treatment $(\triangle \mathrm{BCVA}+0.2)$ compared to the untreated group ( $\triangle \mathrm{BCVA}-0.2, \mathrm{p}=0.15$ ). Likewise statistical significance was missed for the secondary outcome measure. None of the MSON patient received delayed treatment. 


\section{CRION}

There was no statistical significant difference regarding recovery of VA in patients with CRION receiving hyperacute treatment $(\triangle \mathrm{BCVA}-0.1)$ compared to the untreated group ( $\triangle \mathrm{BCVA}-0.2$, $\mathrm{p}=0.43$ ). There was however significantly less pRNFL loss in patients with CRION who received hyperacute treatment $(1.5 \mu \mathrm{m}$ atrophy) if compared to those with delayed treatment $(8 \mu \mathrm{m}$ atrophy, $\mathrm{p}=0.0008$ ). Changes for the mGCL pointed to the same direction but failed statistical significance $(\mathrm{p}=0.12)$ for comparison of the hyperacute treatment group ( $7.8 \mu \mathrm{m}$ atrophy) if compared to those with delayed treatment ( $28 \mu \mathrm{m}$ atrophy).

\section{NMO-ON}

There were only two patients with NMO-ON and no significant differences were observed between these two patients for either the primary or secondary outcome measure. What was however remarkable in this group is that one of the NMO-ON patients (\#10 in Figure 2) who experience a relapse whilst under our care, recovered a BCVA in the affected eye to 1.2 with hyperacute corticosteroid treatment.

\section{Discussion}

This retrospective study suggests that patients with recurrent episodes of optic neuritis have a better visual outcome if corticosteroid treatment is initiated hyperacutely. The outcome was significantly better for both the primary and secondary outcome measures. If corticosteroid treatment was initiated within two days of symptom onset the outcome BCVA was significantly better compared to delayed treatment or no treatment at all. Likewise, hyperacute corticosteroid 
treatment was neuroprotective with better preservation of the mGCL, with atrophy about 12times more severe with delayed treatment initiation.

These data were confirmatory of eight cases published previously [Plant et al. 2011] . A review of the cases published by Plant et al. shows that four cases were treated within two days after symptom onset, one case after four days and for the remaining three cases the delay to treatment initiation was not specified. Taken together half of the case series by Plant et al was treated within two days after symptom onset, which is comparable to the time interval chosen for hyperacute treatment initiation in this study. The case series by Plant et al. did not include serial OCT data [Plant et al. 2011] .

In contrast the ONTT recruited patients within an overall average of five days, permitting for a delay of up to 14 days [Beck et al. 1992b]. A consistent finding between the ONTT and the present study was that delay of treatment within this time frame showed a worse outcome. More specifically the subgroup analysis of present data suggested that the effect of hyperacute treatment on final visual outcome progressively weakened with delay, failing to reach statistical significance if initiated later than five days. There is evidence for manifest axonal degeneration resulting in poor visual outcome within the first two weeks after onset [Petzold,Rejdak \& Plant 2004] .

We would however be hesitant to extrapolate from present findings from patient with recurrent ON to retrospectively interpreting data of a larger population of patients presenting with a first ever episode of ON. Present data suggest are that in patients with recurrent ON treatment 
initiation within two days is preferable to delayed treatment. Biologically early treatment initiation is likely to halt inflammation and prevent axonal degeneration [Zhu et al. 1999] .

Nakamura et al had demonstrated that early corticosteroid treatment in patients with NMO-ON did result in significantly less pRNFL atrophy if compared to patients in whom treatment was delayed [Nakamura et al. 2010] . This finding by Nakamura et al. is consistent with present OCT data.

The current study has a number of important limitations. First, the study design was a retrospective with heterogeneous baseline and follow-up intervals based on clinical practise needs rather than a clinical trial setting. Statistical analysis corrected for heterogeneity, but missing data weakened overall statistical power.

Second, in this study only the effect of corticosteroid therapy was evaluated. All patients with CRION and NMO-ON were on azathioprine and disease modifying treatments were used in MS. Whist there is no evidence that any of these would have had an effect in acute ON occurring under this treatment, such co-medication will need to be factored in future trials, not only for statistics, but also because of ethical concerns regarding placebo controlled trials.

Third, the primary outcome of the current study was the BCVA. This measurement has been used because it was recorded in the clinical routine, although this might not be the most sensitive measure. For instance low contrast visual acuity charts are more sensitive and commonly used in research studies [Balcer,Miller,Reingold \& Cohen 2014] . There needs to be rigorous use of appropriate refraction for sensible collection of low contrast acuity data. Likewise any potential 
future treatment trial on the effect of hyperacute corticosteroids should include visual fields to complement visual acuity.

These criticism aside, a very recent trial has demonstrated the non-inferiority of oral high-dose (1000 mg) methyl-prednisolone for three days compared to IVMP (1000 mg) [Le Page et al. 2015]. The importance of this finding cannot be underestimated because it offers for the first time a realistic option for hyperacute treatment in relapsing optic neuritis.

\section{Conclusion}

This study shows that focusing on recurrent optic neuritis may permit to overcome one of the biggest challenge for optic neuritis treatment trials, namely rapid recruitment. Secondly hyperacute administration of oral corticosteroids, which are non-inferior to IVMP [Le Page et al. 2015] , may, if given hyperacutely, improve visual outcome. The subgroup analysis suggests that this may be of particular relevance for patients with RION, CRION and NMO-ON. Clearly, a prospective study with a rigorous investigation protocol will be needed to investigate this further. For such a study OCT data on the mGCL should be included as a secondary outcome measure [Cruz-Herranz et al. 2016]. The demonstration of hyperacute anti-inflammatory activity of corticosteroids may be further strengthened by inclusion of biomarker data [Moreira,Tilbery,Monteiro,Teixeira \& Teixeira 2006]. Likewise inclusion of biomarkers for axonal degeneration, neurofilament proteins, at presentation will permit to exclude nonresponders because axonal injury as already incurred [Petzold et al. 2004]. 


\section{Methods and materials}

\section{Patients}

A retrospective explorative case review of consecutive patients presenting to a tertiary specialist centre (VUmc, NL) with a diagnosis of ON and optical coherence tomography (OCT) data between January 2010 and January 2014. The study was in accordance with the World Medical Association (WMA) Declaration of Helsinki and national ethics (http://www.ccmo.nl/nl/uwonderzoek-wmo-plichtig-of-niet). The hospital database was searched for the diagnostic codes for "Optic Neuritis" and "Optic Neuropathy".

\section{Inclusion criteria}

The patients had to be known to the centre and have a diagnosis of multiple sclerosis associated ON (MSON), relapsing isolated ON (RION), CRION or NMO-ON made following acute optic neuritis based on established diagnostic criteria [Wingerchuk,Lennon,Pittock, Lucchinetti \& Weinshenker 2006; Polman et al. 2011; Petzold \& Plant 2014; Petzold et al. 2014].

The key difference for distinguishing patients with RION from CRION is that patients with RION experience a spontaneous relapse, whilst patients with CRION will always relapse when taken off immunosuppressive treatment.

Recurrent episodes of ON were defined as at least two episodes of ON affecting either eye [Petzold et al. 2014] . Visual acuity (VA) had to be good enough, $\geq 0.05$ decimals, to permit for 
assessment of longitudinal changes. A baseline OCT scan had to be present. Patients had to be seen in clinic with each attack. Documented loss of vision on presentation lasting for more than 24 hours was required to qualify for treatment, but pain worsening on eye movements was not a mandatory criterion as not present in all cases [Petzold et al. 2014] . For a diagnosis of NMO patients had to be seropositive of AQP4 using a state of the art cell based assay [Wingerchuk et al. 2015] .

\section{Exclusion criteria}

Patients with other causes of visual loss than ON, more than -5 dioptre myopia or transient loss of vision due to Uhthoff phenomenon [Fraser,Davagnanam,Radon \& Plant 2012; Petzold et al. 2014] .

\section{Clinical Assessments}

Patient baseline clinical and demographic characteristics were recorded at baseline. The number of recurrent episodes of $\mathrm{ON}$ was noted. We recorded the best corrected visual acuity (BCVA) using high contrast Snellen charts at presentation and at least three months after a recurrent episode of ON. The change of BCVA was calculated following each recurrent ON. The BCVA was used as the primary outcome measure with worse vision indicating poorer outcome.

\section{Treatment}


The treatment was targeted at the aetiology of recurrent ON. Patients with MSON received methylprednisolone $1000 \mathrm{mg}$ daily for three days. Patients with RION, CRION or NMO-ON had corticosteroid treatment based on their own preference either intravenously with methylprednisolone (1000 mg daily for three days) or orally with prednisolone based individually on what had been the last effective dose during oral taper as borne out during follow-up [Petzold \& Plant 2014] . All patients with CRION and NMO-ON also were on azathioprine as a steroid saving agent [Petzold \& Plant 2014] . Episodes which were not treated with corticosteroids were defined as "no treatment".

\section{Retinal OCT}

The Spectralis OCT (Heidelberg Engineering) was used as a state of the art spectral domain OCT device for all patients included. The peripapillary ring scans and macular volume scans were performed as described [Balk et al. 2014] . To be eligible for data analyses OCT scans had to be available from baseline and prior to each new episode of recurrent $\mathrm{ON}$ and at least three months after onset. All OCT B-scans were quality controlled and reported as recommended [Tewarie et al. 2012; Cruz-Herranz et al. 2016] . All scans were automatically segmented using the Heidelberg Viewing module (version 6.3). Each automatically segmented B-scan was visually checked for integrity and presence of algorithm failures by one author (EO). The averaged peripapillary RNFL (pRNFL), averaged macular ganglion cell layer (mGCL, inner 1-2.2 mm 
circle of EDTRS gird) thickness were calculated. The change of pRNFL and mGCL thickness data between episodes were used as secondary outcome variables, with more atrophy indicating poorer outcome.

\section{Statistical Analysis}

Descriptive statistics were calculated using SAS software (v9.4). Descriptive statistics were presented for normally distributed data as mean and standard deviation (SD). Normality was assessed graphically and using the Shapiro-Wilk test. For non-Gaussian data the median and interquartile range (IQR) were presented. Patients were grouped according to treatment. To enable statistical analysis the Snellen results were converted to decimal values, using a validated conversion table which takes into account vision worse than 6/60 [Petzold et al. 2014] . Data were analysed separately for the affected and unaffected eye per patient and recurrent episode of ON. The time lag from onset to clinical assessment was added as co-variate. Likewise we corrected for age, but not for gender as there were only four male subjects. Consecutively 'hyperacute' groups were composed of episodes started with treatment within two days because sustained loss of vision for at least 24 hours was an inclusion criterion. These 'hyperacute' group was compared with groups who consisted of episodes which received delayed treatment and episodes which received no treatment. Generalised linear models (GLM) were used for comparison. Two tailed-tests were used and p-value of $<0.05$ was accepted as statistically significant. 


\section{Figure legends}

Figure 1. Overview of the patient selection process. In total 40 episodes of recurrent ON occurring in 19 patients had appropriately timed OCT investigations to qualify for inclusion.

Figure 2. Overview episodes per patient and treatment. The number for each patient is shown to the very left of the graph. Patients were grouped clinically into those with MSON (dark shaded grey area), CRION (lighter grey shaded area), NMO (lightest grey shaded area) and RION (white background). For each patient the time from the last episode of recurrent $\mathrm{ON}$ to referral to our centre is shown (number in front of vertical black reference line). At baseline all patients were seen at our centre and entered to the database (vertical black reference line). Recurrent episodes of $\mathrm{ON}$ were indicated at presentation (circle symbol). The treatment received per presentation was and colour coded (red filled circle $=$ no treatment, empty circle $=$ delayed treatment, black filled circle $=$ treatment within 2 days of symptom onset). The cross shows time of last followup.

Figure 3. Change of BCVA following an acute episode of recurrent ON. Patients who received corticosteroids hyperacutely, within two days, had a significantly better recovery of BCVA compared to those with delayed treatment initiation $(\mathrm{p}=0.036)$ or no treatment at all $(\mathrm{p}=0.045)$. 
Figure 4. Change of retinal layer thickness $(\mathrm{mGCL}=$ red bars, $\mathrm{pRNFL}=$ blue bars $)$ following an episode of recurrent ON. The y-axis shows the change of individual retinal layer thickness following an episode of ON. A larger negative value indicates more atrophy. Patients with hyperacute corticosteroid treatment, within two days, had significantly less atrophy of the macular mGCL compared to those with delayed treatment initiation $(\mathrm{p}=0.019)$. Zero $\mathrm{Rx}=$ No treatment, Slow Rx $=$ Delayed treatment, Quick $<2$ Rx $=$ Hyperacute treatment. 


\section{Tables}

Table 1. Baseline characteristics of patients with recurrent episodes of $\mathrm{ON}$ (pooled). $\mathrm{n}=$ number, $\mathrm{IQR}=$ Inter Quartile Range, $\mathrm{M}=$ Male, $\mathrm{F}=$ Female, $\mathrm{RION}=$ relapsing isolated optic neuritis, CRION = Chronic Relapsing Inflammatory Optic Neuropathy, MSON = Multiple Sclerosis associated Optic Neuritis, NMO-ON = Neuromyelitis Optica associated ON.

\begin{tabular}{lll}
\hline Patients, $\mathbf{n}$ & 19 \\
\hline Median age (IQR), years & $31.0(26-44)$ \\
\hline M:F ratio & & $4: 15$ \\
\hline Ethnicity, \% & Caucasian & $90 \%$ \\
& Asian & $5 \%$ \\
& Afro-Caribbean & $5 \%$ \\
\hline Median time follow-up (IQR), Months & $22(13-33)$ \\
\hline AQP4, n & Positive & 2 \\
& Negative & 17 \\
\hline Episodes, $\mathbf{n}$ & \\
Total $\quad$ & 40 \\
\hline Type, $\mathbf{n} \quad$ RION & 9 \\
& CRION & 4 \\
& MSON & 4 \\
NMO-ON & 2 \\
\hline
\end{tabular}


Table 2. Patient characteristics for the clinical subgroups of patients with recurrent ON. There were no statistical differences between these groups for the demographic data. But patients with CRION, RION and NMO-ON had a higher number of recurrent ON compared to those with MSON. Note that in this table the VA is presented separately for the right and left eye, but the figures refer to change of visual acuity in the affected eye.

\begin{tabular}{lllll}
\hline & CRION & RION & MSON & NMO-ON \\
\hline Number of patients, $\mathbf{n}$ & 4 & 9 & 4 & 2 \\
\hline Median Age (IQR), yr & $30.0(22.5-43.5)$ & $40.0(30.0-49.0)$ & $25.5(25.0-34.5)$ & $26.5(26.0-27.0)$ \\
\hline M:F ratio & $1: 3$ & $1: 2$ & $0: 4$ & $0: 2$ \\
\hline $\begin{array}{l}\text { Median VA baseline (IQR), } \\
\text { decimal }\end{array}$ & $0.16(0.002-0.32)$ & $0.45(0.21-0.90)$ & $1.0(0.8-1.0)$ & $0.68(0.10-1.25)$ \\
OD & $0.2(0.2-0.2)$ & $0.8(0.4-1.0)$ & $0.8(0.8-1.2)$ & $1.0(1.0-1.0)$ \\
OS & & & & \\
\hline $\begin{array}{l}\text { Median number of recurrent } \\
\text { ON (IQR) }\end{array}$ & $7.75(3.5-12.0)$ & $5.0(3.0-8.0)$ & $2.0(2.0-5.0)$ & $5.0(3.0-7.0)$ \\
\hline
\end{tabular}


Table 3. Corticosteroid treatment per clinical group. This includes episodes prior to referral to our centre, explaining the lack of treatment of some recurrent episodes in RION and NMO.

\begin{tabular}{llll}
\hline Type & No treatment & Delayed treatment & Hyperacute treatment \\
MSON & $86.0 \%$ & $0.0 \%$ & $14.0 \%$ \\
RION & $25.0 \%$ & $37.5 \%$ & $37.5 \%$ \\
CRION & $0.0 \%$ & $55.0 \%$ & $45.0 \%$ \\
NMO-ON & $33.0 \%$ & $50.0 \%$ & $17.0 \%$ \\
\hline
\end{tabular}




\section{References}

Achiron, A.; Djaldetti, R. and Ziv, I. (1992). Corticosteroids in the treatment of optic neuritis., The New England journal of medicine $327: 281-282$.

Asgari, N.; Owens, T.; Frøkiaer, J.; Stenager, E.; Lillevang, S. T. and Kyvik, K. O. (2011). Neuromyelitis optica (NMO)--an autoimmune disease of the central nervous system (CNS)., Acta Neurol Scand 123 : 369-384.

Balcer, L. J.; Miller, D. H.; Reingold, S. C. and Cohen, J. A. (2014). Vision and vision-related outcome measures in multiple sclerosis, Brain $138: 11-27$.

Balk, L. J.; Twisk, J. W. R.; Steenwijk, M. D.; Daams, M.; Tewarie, P.; Killestein, J.; Uitdehaag, B. M. J.; Polman, C. H. and Petzold, A. (2014). A dam for retrograde axonal degeneration in multiple sclerosis?, Journal of Neurology, Neurosurgery \& Psychiatry 85 : 782-789.

Banik, N. L.; Matzelle, D.; Terry, E. and Hogan, E. L. (1997). A new mechanism of methylprednisolone and other corticosteroids action demonstrated in vitro: inhibition of a proteinase (calpain) prevents myelin and cytoskeletal protein degradation, Brain research 748 : 205-210.

Beck, R. W.; Cleary, P. A.; Anderson Jr, M. M.; Keltner, J. L.; Shults, W. T.; Kaufman, D. I.; Buckley, E. G.; Corbett, J. J.; Kupersmith, M. J.; Miller, N. R. and others (1992a). A randomized, controlled trial of corticosteroids in the treatment of acute optic neuritis, New England Journal of Medicine 326 : 581-588.

Beck, R. W.; Cleary, P. A.; Anderson Jr, M.; Keltner, J. L.; Shults, W. T.; Kaufman, D. I.; Buckley, E. G.; Corbett, J. J.; Kupersmith, M. J. and Miller, N. R. (1992b). A randomized, controlled trial of corticosteroids in the treatment of acute optic neuritis. The Optic Neuritis Study Group., N Engl J Med 326 : 581-588.

Burman, J.; Raininko, R. and Fagius, J. (2011). Bilateral and recurrent optic neuritis in multiple sclerosis., Acta Neurol Scand 123 : 207-210.

Cruz-Herranz, A.; Balk, L. J.; Oberwahrenbrock, T.; Saidha, S.; Martinez-Lapiscina, E. H.; Lagreze, W. A.; Schuman, J. S.; Villoslada, P.; Calabresi, P.; Balcer, L.; Petzold, A.; Green, A. J.; Paul, F.; Brandt, A. U.; Albrecht, P. and consortium , I. (2016). The APOSTEL recommendations for reporting quantitative optical coherence tomography studies., Neurology 86 : 2303-2309.

Fraser, C. L.; Davagnanam, I.; Radon, M. and Plant, G. T. (2012). The time course and phenotype of Uhthoff phenomenon following optic neuritis., Mult Scler 18 : 1042-1044.

Group, O. N. S. and others (1991). The clinical profile of optic neuritis: experience of the Optic Neuritis Treatment Trial, Archives of Ophthalmology $109: 1673$. 
Le Page, E.; Veillard, D.; Laplaud, D. A.; Hamonic, S.; Wardi, R.; Lebrun, C.; Zagnoli, F.; Wiertlewski, S.; Deburghgraeve, V.; Coustans, M.; Edan, G. and others (2015). Oral versus intravenous high-dose methylprednisolone for treatment of relapses in patients with multiple sclerosis (COPOUSEP): a randomised, controlled, double-blind, non-inferiority trial., Lancet 386 : 974-981.

Moreira, M. A.; Tilbery, C. P.; Monteiro, L. P.; Teixeira, M. M. and Teixeira, A. L. (2006). Effect of the treatment with methylprednisolone on the cerebrospinal fluid and serum levels of CCL2 and CXCL1O chemokines in patients with active multiple sclerosis., Acta Neurol Scand 114 : 109-113.

Moskopp, D. (1992). Corticosteroids in the treatment of optic neuritis., The New England journal of medicine $327: 281$; author reply 282.

Nakamura, M.; Nakazawa, T.; Doi, H.; Hariya, T.; Omodaka, K.; Misu, T.; Takahashi, T.; Fujihara, K. and Nishida, K. (2010). Early high-dose intravenous methylprednisolone is effective in preserving retinal nerve fiber layer thickness in patients with neuromyelitis optica., Graefes Arch Clin Exp Ophthalmol 248 : 17771785.

Nikoskelainen, E. (1975). Later course and prognosis of optic neuritis, Acta ophthalmologica 53 : 273 291.

Petzold, A. (2017). Neuroprotection and visual function after optic neuritis., Current opinion in neurology $30: 67-73$.

Petzold, A.; de Boer, J. F.; Schippling, S.; Vermersch, P.; Kardon, R.; Green, A.; Calabresi, P. A. and Polman, C. (2010). Optical coherence tomography in multiple sclerosis: a systematic review and metaanalysis., Lancet Neurol 9 : 921-932.

Petzold, A. and Plant, G. T. (2014). Chronic relapsing inflammatory optic neuropathy: a systematic review of 122 cases reported., J Neurol 261 : 17-26.

Petzold, A.; Rejdak, K. and Plant, G. (2004). Axonal degeneration and inflammation in acute optic neuritis, J Neurol Neurosurg Psychiatry 75 : 1178-1180.

Petzold, A.; Wattjes, M. P.; Costello, F.; Flores-Rivera, J.; Fraser, C. L.; Fujihara, K.; Leavitt, J.; Marginier, R.; Paul, F.; Schippling, S.; Sindic, C.; Villoslada, P.; Weinshenker, B. and Plant, G. T. (2014). The investigation of acute optic neuritis: a review and proposed protocol., Nat Rev Neurol 10 : 447-458.

Plant, G. T.; Sibtain, N. A. and Thomas, D. (2011). Hyperacute corticosteroid treatment of optic neuritis at the onset of pain may prevent visual loss: a case series., Mult Scler Int $2011: 815068$.

Polman, C. H.; Reingold, S. C.; Banwell, B.; Clanet, M.; Cohen, J. A.; Filippi, M.; Fujihara, K.; Havrdova, E.; Hutchinson, M.; Kappos, L.; Lublin, F. D.; Montalban, X.; OĆonnor, P.; Sandberg-Wollheim, M.; Thompson, A. J.; Waubant, E.; Weinshenker, B. and Wolinsky, J. S. (2011). Diagnostic criteria for multiple sclerosis: 2010 revisions to the McDonald criteria., Ann Neurol 69 : 292-302. 
Rosenberg-Schaffer, L. J. and Lucas, J. H. (1993). An in vitro study of the effects of methy/prednisolone on lesioned and uninjured mammalian spinal neurons, Brain research 605 : 327-331.

Sapolsky, R. M.; Romero, L. M. and Munck, A. U. (2000). How do glucocorticoids influence stress responses? Integrating permissive, suppressive, stimulatory, and preparative actions 1 , Endocrine reviews $21: 55-89$.

Shindler, K. S.; Ventura, E.; Dutt, M. and Rostami, A. (2008). Inflammatory demyelination induces axonal injury and retinal ganglion cell apoptosis in experimental optic neuritis, Experimental eye research 87 : 208-213.

Tewarie, P.; Balk, L.; Costello, F.; Green, A.; Martin, R.; Schippling, S. and Petzold, A. (2012). The OSCARIB Consensus Criteria for Retinal OCT Quality Assessment., PLoS One 7 : e34823.

Wingerchuk, D. M.; Banwell, B.; Bennett, J. L.; Cabre, P.; Carroll, W.; Chitnis, T.; de Seze, J.; Fujihara, K.; Greenberg, B.; Jacob, A.; Jarius, S.; Lana-Peixoto, M.; Levy, M.; Simon, J. H.; Tenembaum, S.; Traboulsee, A. L.; Waters, P.; Wellik, K. E. and Weinshenker, B. G. (2015). International consensus diagnostic criteria for neuromyelitis optica spectrum disorders., Neurology .

Wingerchuk, D. M.; Lennon, V. A.; Pittock, S. J.; Lucchinetti, C. F. and Weinshenker, B. G. (2006). Revised diagnostic criteria for neuromyelitis optica., Neurology 66 : 1485-9.

Zhu, B.; Moore, G. W.; Zwimpfer, T. J.; Kastrukoff, L. F.; Dyer, J. K.; Steeves, J. D.; Paty, D. W. and Cynader, M. S. (1999). Axonal cytoskeleton changes in experimental optic neuritis, Brain research 824 : 204-217. 


\section{Highlights}

- Optic neuritis treatment trials have gained new relevance for testing neuroprotective and remyelination treatment strategies in demyelinating disease.

- Rapid recruitment of patients with a first episode of optic neuritis remains a big challenge for optic neuritis treatment trials

- Recurrent optic neuritis may offer a chance for rapid recruitment as patients are already known to the service

- Time is vision

- This retrospective study suggests that rapid patient recruitment to trials is possible with recurrent optic neuritis 
Supplementary Figure : The BCVA of the symptomatic eye (large gray shaded bars) and fellow eye (small dotted bars) per treatment group at subsequent time points; $(A)$. within three, (B) four, (C) five, (D) six days and (E) comparable to the ONTT after 14 days after symptom onset.

The mean and standard deviation are shown. P- value for treatment groups of affected eyes. Zero Rx $=$ No treatment. Slow Rx $=$ Delayed treatment, Quick Rx $=$ Hyperacute treatment.
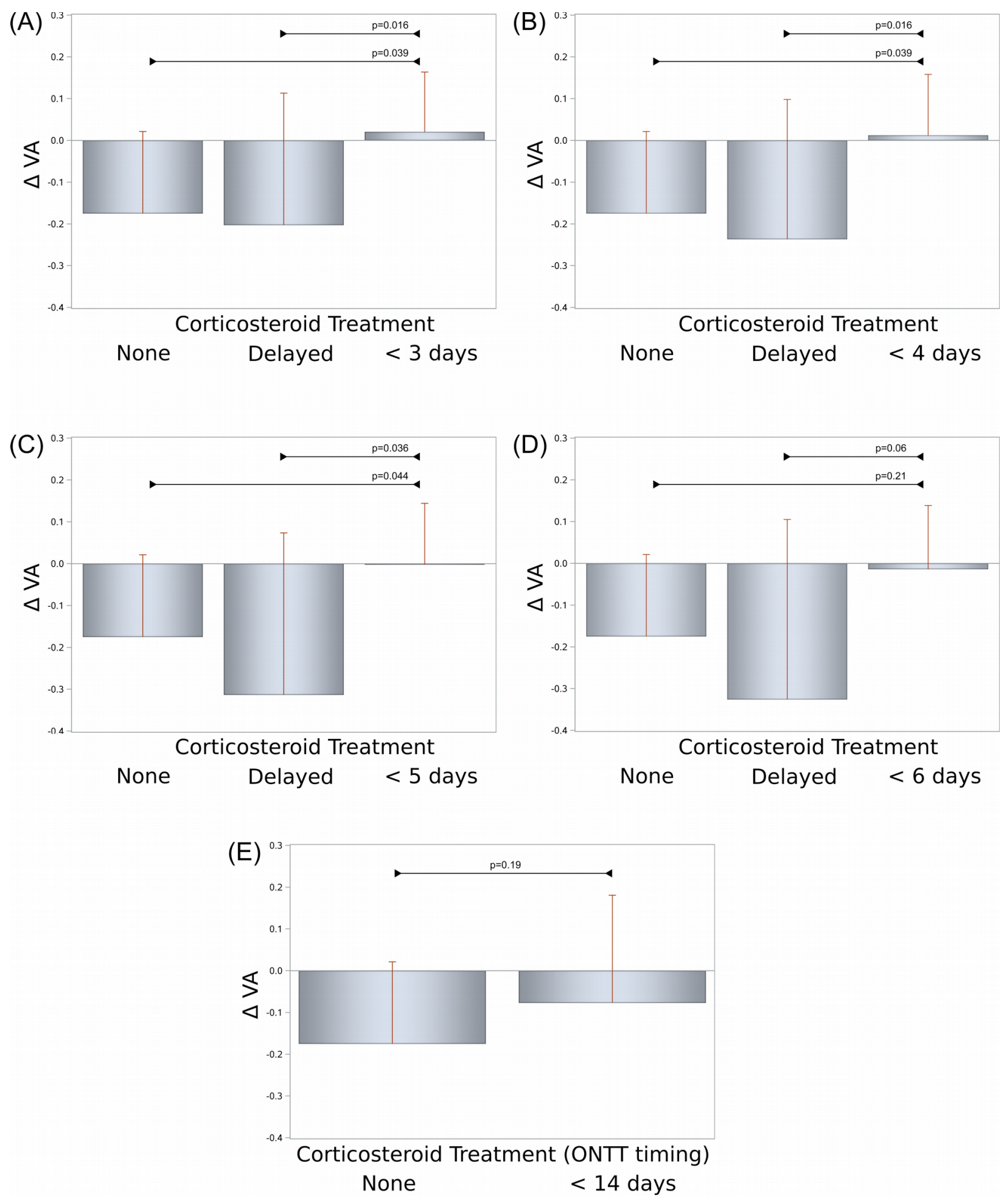
Assessed for DBC/DOT-

code "Optic Neuritis" and "Optic Neuropathy"

Assessed if OCT-scan was present $(n=269)$

No OCT-scan present $(n=168)$

Investigated for accurate diagnosis ON $(n=101)$

\section{No diagnosis ON $(n=47)$}

Assessed for number of episodes of ON $(n=54)$

Included $(n=19)$, consisting of 40 episodes of ON 


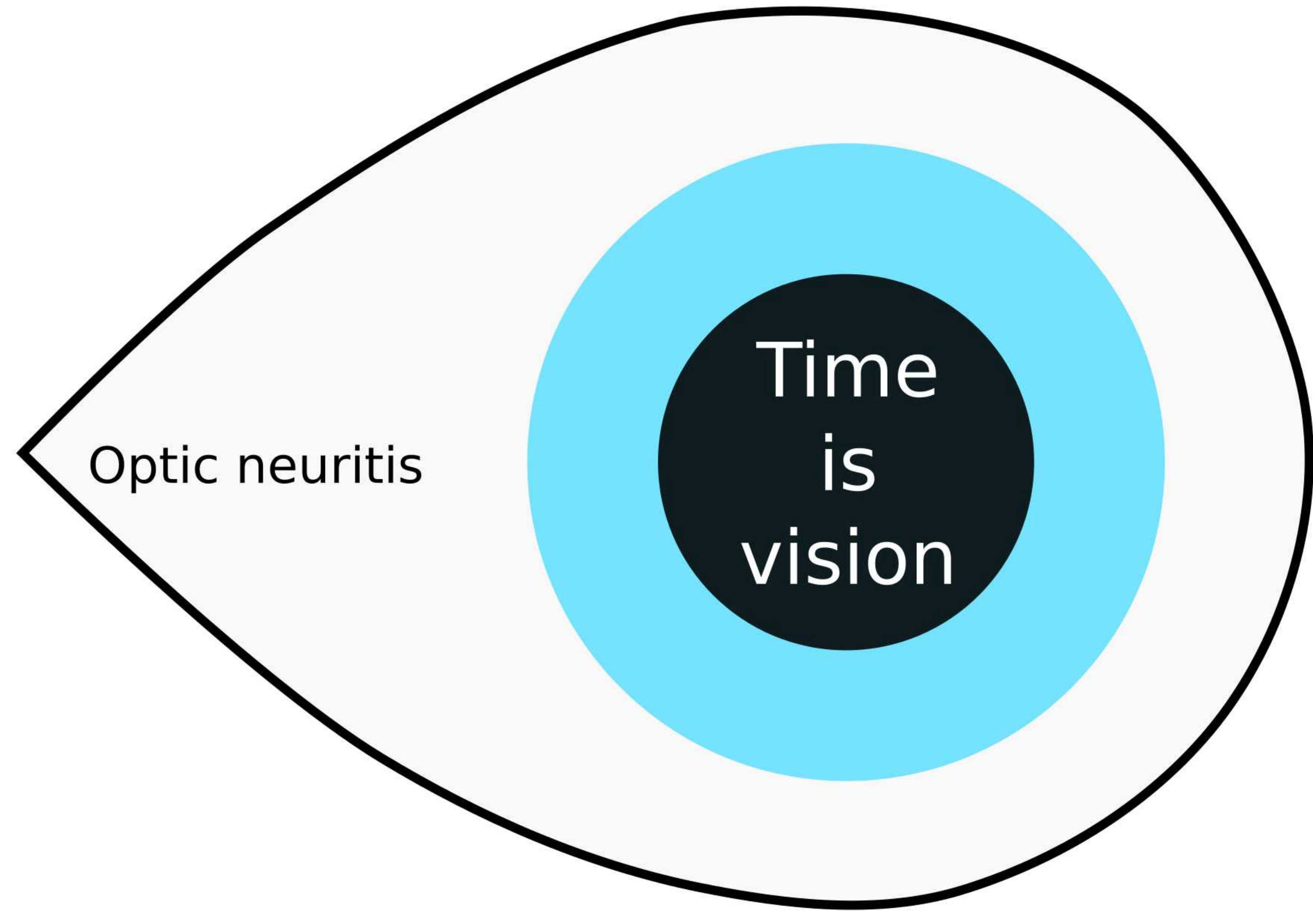




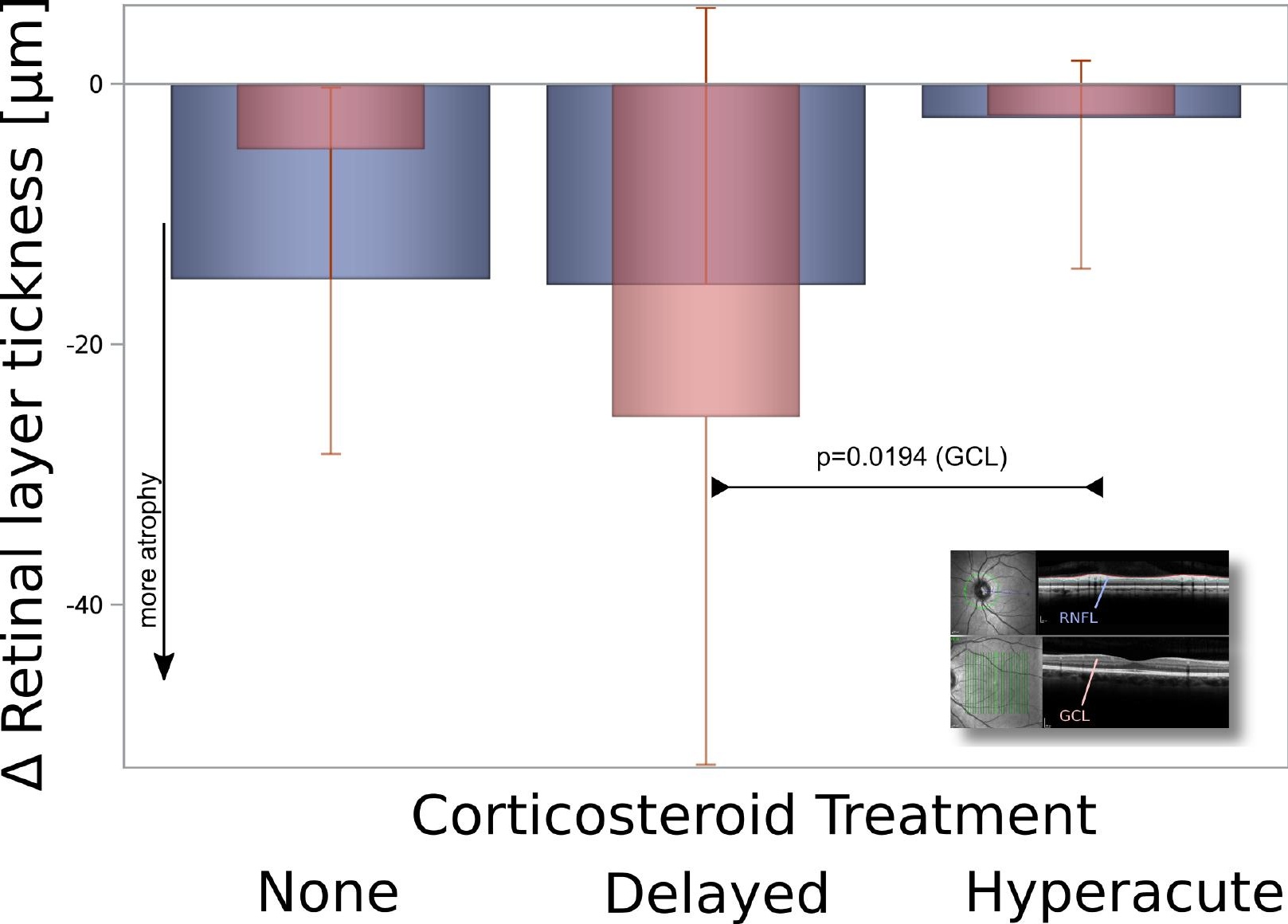


